\title{
Evaluation of Chronic Liver Disease: Does Ultrasound Scoring Criteria Help?
}

\author{
Shaista Afzal, Imrana Masroor, and Madiha Beg \\ Aga Khan University Hospital (AKUH), Stadium road, Karachi 74800, Pakistan \\ Correspondence should be addressed to Shaista Afzal; shaista.afzal@aku.edu
}

Received 31 May 2013; Accepted 25 July 2013

Academic Editor: Maria Gazouli

Copyright ( 2013 Shaista Afzal et al. This is an open access article distributed under the Creative Commons Attribution License, which permits unrestricted use, distribution, and reproduction in any medium, provided the original work is properly cited.

\begin{abstract}
Noninvasive approaches for assessment of liver histology include routine laboratory tests and radiological evaluation. The purpose of our study was to determine the utility of a simplified scoring system based on routinely evaluated ultrasound features for the evaluation of chronic liver disease and correlate it with the histological findings. For this cross-sectional analytical study the data was collected prospectively by nonprobability purposive sampling technique. The ultrasound variables/parameters and their assigned scoring system that was a modified version adopted from published literature were evaluated. Sensitivity, specificity, positive and negative predictive values of the liver morphological score and combined score of liver morphology and sizes was determined using stage and grade as reference standard. Our results show a high sensitivity and PPV of liver morphological sonographic evaluation for the staging and grading of CLD respectively thus supporting it as a screening diagnostic strategy. Of the three liver morphology variables, specificity of liver surface evaluation was highest for the stage of fibrosis and grade of inflammation. The simplified ultrasound scoring system evaluated in our study is clinically relevant and reproducible for differentiating patients with CLD with mild or no fibrosis from moderate to severe fibrosis.
\end{abstract}

\section{Introduction}

The common causes of chronic liver disease are viral hepatitis, alcohol abuse, and metabolic disorders. These result in hepatocytes damage, the consequence of which may be liver fibrosis, cirrhosis, and/or hepatocellular carcinoma [1]. The disease is a substantial cause of morbidity and mortality in the developing countries. Accurate evaluation of the severity of disease is crucial for treatment planning, that is, commencement of antiviral treatment and prognostication [2].

Noninvasive approaches for assessment of liver histology include routine laboratory tests like serum markers, liver functions test, and radiological evaluation of liver. Liver histological diagnosis based on needle biopsy determines the inflammatory activity (grading), the extent of fibrosis (staging), and other comorbidities [3]. But the procedure of ultrasound guided liver biopsy is invasive with about $1 \%$ risk of significant complications like postinterventional hemorrhage, bile leak, infection, and injury to adjacent organs with less than $0.1 \%$ mortality [4]. Sampling errors may also be encountered since the liver parenchymal damage in chronic hepatitis is not homogeneous. In addition there is possibility of inter- and intraobserver variability [5]. Imaging technologies, particularly ultrasound, are inexpensive, noninvasive, readily available, and acceptable to the patient. It is routinely utilized in evaluation of spectrum of chronic liver disease as it provides useful information on the morphological alterations of the liver and organs affected as a result of portal hypertension; in addition color Doppler flow imaging provides information regarding the liver hemodynamics. The other imaging modalities like computerized tomography (CT) and magnetic resonance imaging (MRI) are also helpful, but these are expensive and require contrast administration [6]. A number of ultrasound variables based on liver morphology, hemodynamics, and different techniques of ultrasound like simultaneous use of high and low frequency transducers have been evaluated to predict the liver fibrosis stage with variable accuracy. The purpose of our study was to determine the utility of a simplified scoring system based on routinely evaluated ultrasound features for the evaluation of chronic liver disease and correlate it with the histological findings. 
TABLE 1: Ultrasound variable and scores.

\begin{tabular}{lccc}
\hline Variables & Score 0 & Score 1 & Score 2 \\
\hline Liver parenchymal echotexture & Homogenous/fine & Coarse & Highly nonhomogenous/coarse \\
Liver surface & Smooth & Irregular & Nodular \\
Liver edge (inferior margin right lobe) & Sharp (acute) & Blunted & Rounded \\
Liver size & Normal & Enlarged $(>15 \mathrm{~cm} \mathrm{mid}$ & Shrunken $(<10 \mathrm{~cm}$ in mid \\
clavicular line $)$
\end{tabular}

The concept is to evolve an effective, simple to understand, applicable, and radiologically relevant scoring system for evaluation of the extent of chronic liver disease.

\section{Material and Methods}

This cross-sectional analytical study was performed in the Department of Radiology AKUH from January 2010 to December 2011. The data was collected prospectively by nonprobability purposive sampling technique. All patients sent to the Radiology Department of Aga Khan University Hospital for ultrasound guided liver biopsy were included. Patients were excluded if the histopathology report of liver biopsy was not available and if the biopsy was performed for focal lesions or autoimmune liver disease. In addition patients unfit for liver biopsy due to jaundice, ascites, and deranged blood profiles were also excluded. Patients' confidentiality was guaranteed and maintained during the course of the study.

Prior to the biopsy real time ultrasound using Toshiba Nemio XG was performed for all patients using 3.5-5.0 MHz convex transducer by the radiologist on duty in the ultrasound interventional suit having at least 3 years of experience in performing abdominal sonography. Ultrasound of the liver was performed, both lobes of liver were evaluated, and a combined impression was derived. In addition size of liver, spleen, and portal vein was also assessed and noted. The ultrasound parameters and scoring system were explained to examining radiologist prior to the procedure and findings recorded on a standard proforma. The ultrasound variables/parameters and their assigned scoring system that was a modified version adopted from the published literature are as depicted in Table $1[7,8]$.

The sample for the liver biopsy was obtained from the right lobe of liver-anterior segment using Bards Tru-Cut Biopsy 18 gauge needle. The specimen was reviewed by histopathologist unaware of the ultrasound findings.

The histopathology reports were reviewed through the hospital information system and assessed for grading and staging of the biopsy specimen which was analyzed using the Batts and Ludwig scoring system. Grade evaluated the degree and location of inflammation and stage assessing the location and extent of fibrosis in the biopsy specimen. According to the histopathology scoring system stage 0 was described as no fibrosis and with increasing fibrosis a score of 4 was assigned for cirrhosis. For the grading score 0 described portal inflammation only and with increasing lobular inflammation and necrosis, score of 4 denoting severe diffuse hepatocellular damage with bridging necrosis [9].

For the purpose of analysis stage and grade 0 and 1 were taken as mild or no disease and stages 2,3 , and 4 as moderate to severe disease.

The ultrasound scoring system was also categorized as "A" for liver morphological evaluation comprising of liver surface, parenchymal echo texture, and edge and "B" for the combined score of liver morphology as detailed above and sizes evaluation of liver, spleen, and portal vein.

2.1. Sample Size Calculation. Calculated sensitivity of ultrasound for detecting chronic liver disease is 77\% [10] with confidence level of $95 \%$, margin of error $10 \%$, and calculated sample size $N=115$. This was done by using the following formula $N=\left[z_{1-\alpha / \varepsilon}^{2}\left(1-P / \varepsilon^{2} P\right)\right]$.

2.2. Plan of Analysis. Data was entered and analyzed using SPSS windows package version 19.0. Frequencies were calculated, and proportions reported for categorical variables. Mean and standard deviations calculated for quantitative variable like age. Sensitivity, specificity, positive, and negative predictive values with $95 \%$ confidence intervals were reported for ultrasound in detecting chronic liver disease in the patients taking histopathology/biopsy as gold standard.

\section{Results}

The study population ( $N=116)$ included predominantly males, $74(64 \%)$ with a mean age of 39.54 years \pm SD 12.77, range between 15 and 70 years. Data was collected of 116 patients prospectively over a period of two years from a tertiary care center, the Aga Khan University Hospital. Out of the 116 patients, 78 (67\%) were hepatitis $C$ reactive.

Sensitivity, specificity, positive and negative predicted values of the liver morphological score denoted as "A" and combined score of liver morphology and sizes denoted as " $\mathrm{B}$ " was determined using stage and grade as reference standard (Table 2).

Majority of patients 97 (84\%) presented with normal liver size, 11 (9\%) presented with an enlarged liver, and $8(7 \%)$ presented with a liver smaller in size. The liver surface was smooth in $71(61 \%)$, while 32 (28\%) showed a mildly irregular liver surface. $13(11 \%)$ presented with an irregular liver surface. 
TABLE 2: Accuracy of US scoring system.

\begin{tabular}{|c|c|c|c|c|c|}
\hline & Histopathology & Sensitivity & Specificity & PPV & NPV \\
\hline \multirow{2}{*}{$\mathrm{A}=$ liver morphological score } & Stage & $90.3 \%$ & $47.7 \%$ & $73.9 \%$ & $75 \%$ \\
\hline & Grade & $84.1 \%$ & $44.1 \%$ & $78.4 \%$ & $53.6 \%$ \\
\hline \multirow{2}{*}{$\begin{array}{l}\mathrm{B}=\text { liver morphological score }+ \\
\text { liver, spleen, and portal vein size }\end{array}$} & Stage & $44.4 \%$ & $88.6 \%$ & $86.5 \%$ & $49.4 \%$ \\
\hline & Grade & $41.5 \%$ & $91.2 \%$ & $92 \%$ & $39.2 \%$ \\
\hline
\end{tabular}

TABLE 3: (a) Diagnostic performance of US variables in predicting fibrosis/stage. (b) Diagnostic performance of US variables in predicting grade.

(a)

\begin{tabular}{lccccc}
\hline Variable & Sensitivity $\left(95 \% \mathrm{CI}^{*}\right)$ & Specificity $\left(95 \% \mathrm{CI}^{*}\right)$ & ${ }^{\wedge} \mathrm{PPV}\left(95 \% \mathrm{CI}^{*}\right)$ & ${ }^{\circledR} \mathrm{NPV}\left(95 \% \mathrm{CI}^{*}\right)$ & ${ }^{\mathrm{a}} P$ value \\
\hline Liver edge & $84.72(0.74,0.92)$ & $61.36(0.46,0.75)$ & $78.21(0.67,0.86)$ & $71.05(0.55,0.84)$ & 0.000 \\
Liver surface & $54.16(0.42,0.65)$ & $86.36(0.72,0.94)$ & $86.66(0.72,0.94)$ & $53.52(0.41,0.65)$ & 0.000 \\
Liver texture & $75(0.63,0.84)$ & $66(0.49,0.79)$ & $78.26(0.66,0.86)$ & $61.7(0.46,0.75)$ & 0.000 \\
Liver size & $12.5(0.06,0.22)$ & $77.27(0.61,0.88)$ & $47.3(0.25,0.70)$ & $35.05(0.26,0.45)$ & 0.149 \\
Spleen size & $9.72(0.04,0.19)$ & $93.2(0.80,0.98)$ & $70(0.35,0.91)$ & $38.68(0.29,0.48)$ & 0.589 \\
Portal vein diameter & $5.55(0.01,0.12)$ & $100^{(\infty)}$ & $100^{(\infty)}$ & $39.28(0.30,0.48)$ & 0.112 \\
\hline
\end{tabular}

${ }^{\wedge} \mathrm{PPV}$ : positive predictive value.

${ }^{\S} \mathrm{NPV}$ : negative predictive value.

$\left(95 \% \mathrm{CI}^{*}\right)$ : $95 \%$ confidence internal.

${ }^{\text {x }} P$ value: chi-square test $P$ value.

${ }^{(\infty)}$ : Cannot be calculated because either of one cell contains a "zero."

(b)

\begin{tabular}{lccccc}
\hline Variable & Sensitivity $\left(95 \% \mathrm{CI}^{*}\right)$ & Specificity $\left(95 \% \mathrm{CI}^{*}\right)$ & ${ }^{\wedge} \mathrm{PPV}\left(95 \% \mathrm{CI}^{*}\right)$ & ${ }^{\S} \mathrm{NPV}\left(95 \% \mathrm{CI}^{*}\right)$ & ${ }^{\mathrm{a}} P$ value \\
\hline liver edge & $80.5(0.70,0.88)$ & $64.71(0.46,0.79)$ & $84.62(0.74,0.91)$ & $57.89(0.41,0.73)$ & 0.000 \\
liver surface & $51.22(0.40,0.62)$ & $91.18(0.75,0.97)$ & $93.33(0.81,0.98)$ & $43.66(0.32,0.55)$ & 0.000 \\
liver texture & $72(0.61,0.81)$ & $70.6(0.52,0.84)$ & $85.51(0.74,0.92)$ & $51.06(0.36,0.65)$ & 0.000 \\
liver size & $14.63(0.08,0.24)$ & $79.41(0.61,0.90)$ & $63.16(0.38,0.82)$ & $27.83(0.19,0.38)$ & 0.430 \\
spleen size & $9.76(0.04,0.18)$ & $94.12(0.78,0.98)$ & $80(0.44,0.96)$ & $30.28(0.22,0.39)$ & 0.499 \\
portal vein diameter & $4.88(0.01,0.12)$ & $100^{(\infty)}$ & $100^{(\infty)}$ & $30.36(0.22,0.39)$ & 0.190 \\
\hline
\end{tabular}

${ }^{\wedge} \mathrm{PPV}$ : positive predictive value.

${ }^{\S} \mathrm{NPV}$ : negative predictive value.

(95\% $\mathrm{CI}^{*}$ ): $95 \%$ confidence internal.

${ }^{\mathrm{g}} P$ value: chi-square test $P$ value.

${ }^{(\infty)}$ : Cannot be calculated because either of one cell contains a "zero."

Liver edge was sharp in 38 (33\%), mildly blunted in 66 (57\%), and rest $12(10 \%)$ showed a blunted liver edge. Portal vein was normal in 112 (97\%) and dilated in the remaining of the total sample.

Sensitivity, specificity, positive and negative predicted values and $P$-value using chi-square test of the each ultrasound variable were determined using stage and grade as reference standard. \{Tables 3(a) and 3(b)\}.

\section{Discussion}

Chronic liver disease is a spectrum of disease manifestation leading to cirrhosis. Current development and improvement in the treatment and management options have stressed a need for prompt diagnosis of CLD to identify asymptomatic patients in a population that is high risk, for example, due to high prevalence of viral hepatitis, and hence provide a better patient outcome. Accurate estimation of the degree of hepatic damage in fibrosis or cirrhosis before the compensation becomes clinically evident is crucial for treatment, prognosis, and surveillance. The noninvasive methods to assess features of CLD include serologic fibrosis markers like fibro test, aspartate aminotransferase-to-platelet ratio index (APRI), and radiologic imaging [11]. These tests are regarded to be perfect and ideal only if these are simple, accessible cheap, and exhibit high accuracy.

In the present study we attempted to develop a simplified scoring system based on ultrasound parameters routinely evaluated in sonographic studies and likely to be affected during the course of CLD like liver morphological appearance and the dimension of liver, portal vein, and spleen. The US scores were compared with the histopathological results of the biopsy specimen. A number of studies have utilized the ultrasound examination for the diagnosis and staging of chronic liver disease making use of different techniques like the conventional gray scale and Doppler $[12,13]$ to 
TABLE 4: Comparison with prior studies of validity of ultrasound score in the evaluation of chronic liver disease.

\begin{tabular}{|c|c|c|c|c|}
\hline Study & Number of patients & Characteristics & Sensitivity & Specificity \\
\hline Gaiani et al. [13] 1997 & 212 & $\begin{array}{l}\text { US scoring comprising of } \\
\text { seven morphological and } \\
\text { hemodynamic hepatic } \\
\text { parameters }\end{array}$ & $82 \%$ & $79 \%$ \\
\hline Hung et al. [8] 2003 & 210 & $\begin{array}{l}\text { US scoring comprising of } \\
\text { liver surface, parenchyma, } \\
\text { vascular structure, and } \\
\text { spleen size }\end{array}$ & $\begin{array}{l}\text { HBV-related cirrhosis } \\
77.8 \% \\
\text { HCV-related cirrhosis } \\
82.4 \%\end{array}$ & $\begin{array}{l}\text { HBV-related cirrhosis } \\
92.5 \% \\
\text { HCV-related cirrhosis } \\
70.7 \%\end{array}$ \\
\hline Choong et al. [15] 2012 & 156 & $\begin{array}{l}\text { Three hepatic features } \\
\text { assessed }\end{array}$ & $\begin{array}{l}53 \% \text { (combined score liver } \\
\text { surface and edge) }\end{array}$ & $\begin{array}{l}94 \% \text { (combined score of } \\
\text { surface and texture) }\end{array}$ \\
\hline Afzal et al. 2013 & 116 & $\begin{array}{l}\text { US scoring system based on } \\
\text { six variables }\end{array}$ & $\begin{array}{l}\text { Liver morphology } 90.3 \% \\
\text { (stage) } \\
84.1 \% \text { (grade) } \\
\text { Liver morphology }+ \text { size of } \\
\text { liver, spleen, and PV } \\
44.4 \% \text { (stage) } \\
41.5 \% \text { (grade) }\end{array}$ & $\begin{array}{l}\text { Liver morphology } 47.7 \% \\
\text { (stage) } \\
44.1 \% \text { (grade) } \\
\text { Liver morphology }+ \text { size of } \\
\text { liver, spleen, and PV } \\
88.6 \% \text { (stage) } \\
91.2 \% \text { (grade) }\end{array}$ \\
\hline
\end{tabular}

sophisticate technique of transient elastography and using contrast agent $[11,14]$. Gaiani et al. [13] investigated patient with chronic liver disease for the presence of compensated cirrhosis using ultrasound scoring system and achieved the sensitivity and specificity of $78.7 \%$ and $80.2 \%$, respectively. A comparison of prior studies using ultrasound scores for the evaluation of chronic liver disease is shown in Table 4.

Our results show a high sensitivity and PPV of liver morphological sonographic evaluation for the staging and grading of CLD, respectively, thus supporting it as a screening diagnostic strategy. The two groups of liver fibrosis that is mild/no fibrosis and moderate/severe fibrosis/cirrhosis could be differentiated using this scoring system with high sensitivity and PPV. This is likely to be related to the fact that the simplified scoring system in the present study evaluated the findings on a 3 level scale, that is, 0,1 , and 2 as compared to other studies [15] where findings were evaluated on a 4-point scale ranging from 0 to 4 .

Of the three liver morphology variables, liver surface evaluation depicted specificity of $86.3 \%$ for the stage of fibrosis and $91.1 \%$ for the grade of inflammation. The result is in keeping with other studies that showed a high specificity of surface nodularity [16]. In this prospective study liver edge was also found to have a high sensitivity and specificity for detection of liver fibrosis and grades of inflammation and differed from other studies in which liver edge was not found to be specific for liver fibrosis evaluation [7].

In this study the cut-off value of the ultrasound score was 2 for liver morphology (category A) and 3 for combination of morphology and sizes (category B). The liver morphology score using 3 variables provided a sensitivity of $90.3 \%$, but a sensitivity of $44.4 \%$ was achieved when all 6 variables were assessed and is lower than that reported by using 4 variables [8]. The patients with clinically decompensated CLD were excluded in the present study to maximize the efficacy of the ultrasound examination. But in addition to the US signs for assessing liver parenchyma, signs consistent with advanced liver disease like enlarged spleen, shrunken liver, and portal hypertension were also evaluated for their presence in nonsymptomatic patients. The number of patients diagnosed as stage IV fibrosis on histopathology in the present study is $19(16.4 \%)$, while on sonography the frequency of small shrunken liver and splenomegaly is $8(7 \%)$ and $10(8.6 \%)$, respectively.

This study has a few limitations. The study results show high sensitivity, but the specificity is low, and hence there is a need to come up with further research to get better diagnostic accuracy. This can be achieved by addressing factors such as intra and inter observer variability, quality assurance of the technique and equipment of ultrasound. Since liver histology was taken as gold standard in this study, the possibility of sampling errors and inter- and intraobserver variability in assessment of biopsy specimen cannot be ruled out and may have also affected our results.

Presence of hepatic steatosis significantly affects the liver parenchymal appearance, but this finding was not assessed in the US evaluation of the study group.

\section{Conclusion}

The simplified ultrasound scoring system evaluated in our study is clinically relevant and reproducible for differentiating patients with CLD with mild or no fibrosis to moderate to severe fibrosis. Since we also evaluated the sensitivity of these parameters for grading, it is also helpful in determining the prognosis and best possible therapeutic option.

\section{References}

[1] D. Schuppan and N. H. Afdhal, "Liver cirrhosis," The Lancet, vol. 371, no. 9615, pp. 838-851, 2008.

[2] R. Zheng, Q. Wang, M. Lu et al., "Liver fibrosis in chronic viral hepatitis: an ultrasonographic study," The World Journal of Gastroenterology, vol. 9, no. 11, pp. 2484-2489, 2003. 
[3] E. M. Brunt, "Grading and staging the histopathological lesions of chronic hepatitis: the Knodell histology activity index and beyond," Hepatology, vol. 31, no. 1, pp. 241-246, 2000.

[4] D. C. Rockey, S. H. Caldwell, Z. D. Goodman, R. C. Nelson, and A. D. Smith, "Liver biopsy," Hepatology, vol. 49, no. 3, pp. 10171044, 2009.

[5] S. Mueller, G. Millonig, L. Sarovska et al., "Increased liver stiffness in alcoholic liver disease: differentiating fibrosis from steatohepatitis," The World Journal of Gastroenterology, vol. 16, no. 8, pp. 966-972, 2010.

[6] H. Tchelepi, P. W. Ralls, R. Radin, and E. Grant, "Sonography of diffuse liver disease," Journal of Ultrasound in Medicine, vol. 21, no. 9, pp. 1023-1034, 2002.

[7] T. Nishiura, H. Watanabe, M. Ito et al., "Ultrasound evaluation of the fibrosis stage in chronic liver disease by the simultaneous use of low and high frequency probes," British Journal of Radiology, vol. 78, no. 927, pp. 189-197, 2005.

[8] C. Hung, S. Lu, J. Wang et al., "Correlation between ultrasonographic and pathologic diagnoses of hepatitis B and C virusrelated cirrhosis," Journal of Gastroenterology, vol. 38, no. 2, pp. 153-157, 2003.

[9] S. B. Rahn, "Liver biopsy interpretation in chronic hepatitis," Journal of Insurance Medicine, vol. 33, no. 1, pp. 110-113, 2001, Review.

[10] A. E. A. Joseph, S. H. Saverymuttu, S. Al-Sam, M. G. Cook, and J. D. Maxwell, "Comparison of liver histology with ultrasonography in assessing diffuse parenchymal liver disease," Clinical Radiology, vol. 43, no. 1, pp. 26-31, 1991.

[11] M. Friedrich-Rust, K. Wunder, S. Kriener et al., "Liver fibrosis in viral hepatitis: noninvasive assessment with acoustic radiation force impulse imaging versus transient elastography," Radiology, vol. 252, no. 2, pp. 595-604, 2009.

[12] Y. Xu, B. Wang, and H. Cao, "An ultrasound scoring system for the diagnosis of liver fibrosis and cirrhosis," Chinese Medical Journal, vol. 112, no. 12, pp. 1125-1128, 1999.

[13] S. Gaiani, L. Gramantieri, N. Venturoli et al., "What is the criterion for differentiating chronic hepatitis from compensated cirrhosis? A prospective study comparing ultrasonography and percutaneous liver biopsy," Journal of Hepatology, vol. 27, no. 6, pp. 979-985, 1997.

[14] J. Searle, R. Mendelson, M. Zelesco et al., "Non-invasive prediction of the degree of liver fibrosis in patients with hepatitis $\mathrm{C}$ using an ultrasound contrast agent. A pilot study," Journal of Medical Imaging and Radiation Oncology, vol. 52, no. 2, pp. 130133, 2008.

[15] C. C. Choong, S. K. Venkatesh, and E. P. Siew, "Accuracy of routine clinical ultrasound for staging of liver fibrosis," Journal of Clinical Imaging Science, vol. 2, article 58, 2012.

[16] A. Colli, M. Fraquelli, M. Andreoletti, B. Marino, E. Zuccoli, and D. Conte, "Severe liver fibrosis or cirrhosis: accuracy of US for detection-analysis of 300 cases," Radiology, vol. 227, no. 1, pp. 89-94, 2003. 


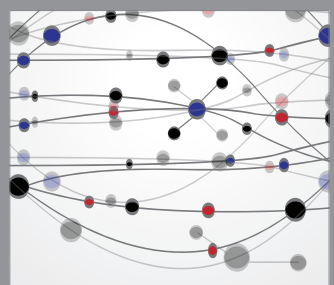

The Scientific World Journal
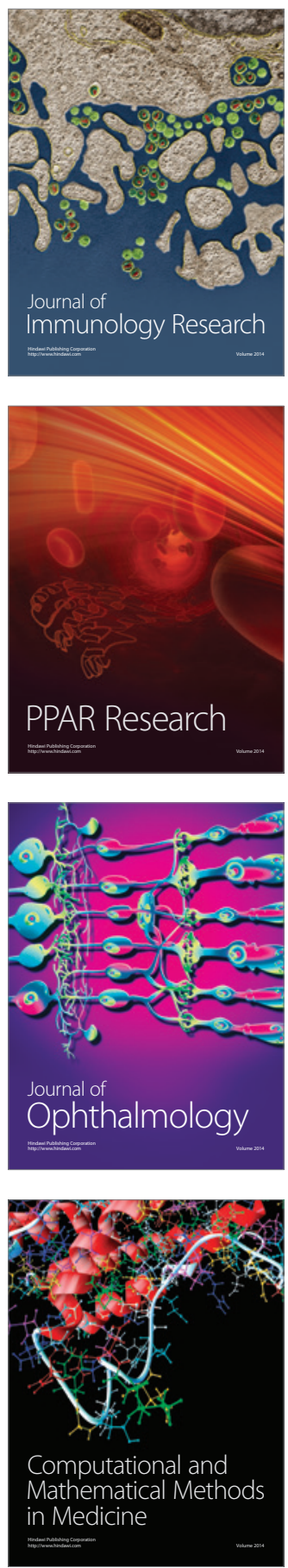

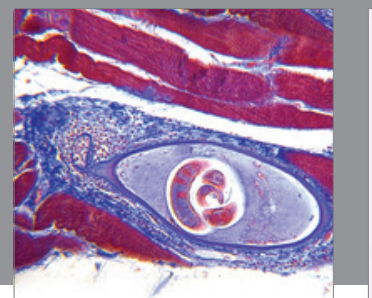

Gastroenterology

Research and Practice
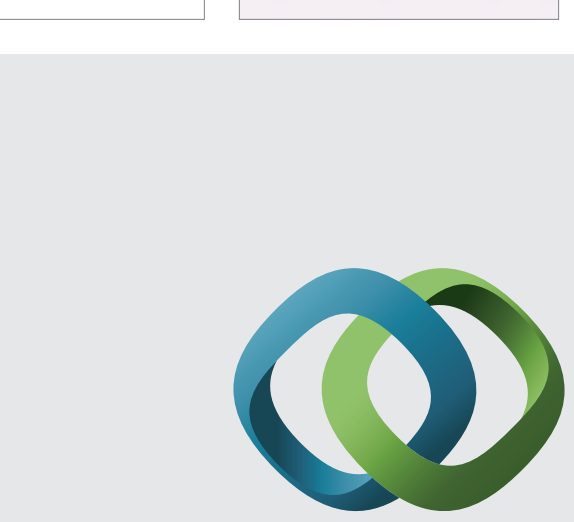

\section{Hindawi}

Submit your manuscripts at

http://www.hindawi.com
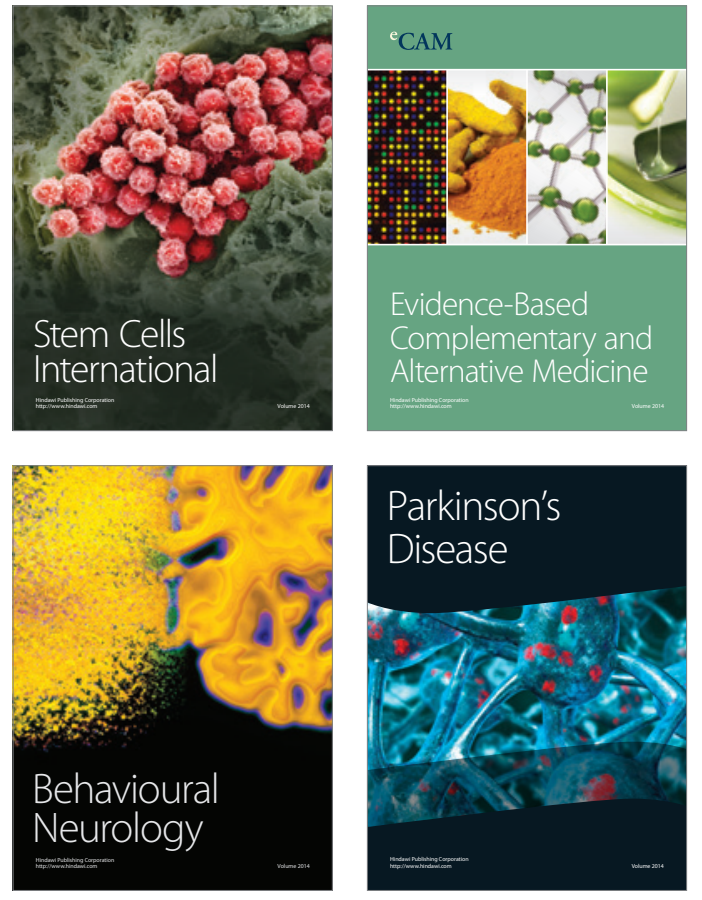
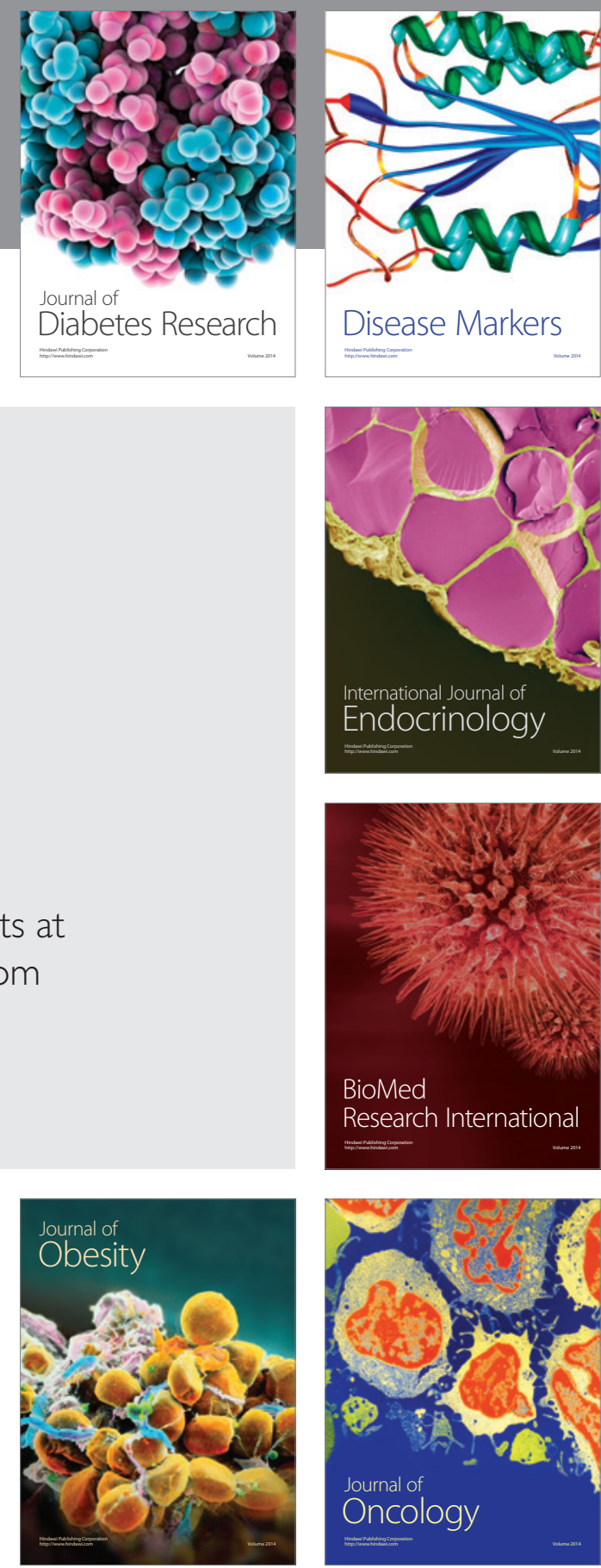

Disease Markers
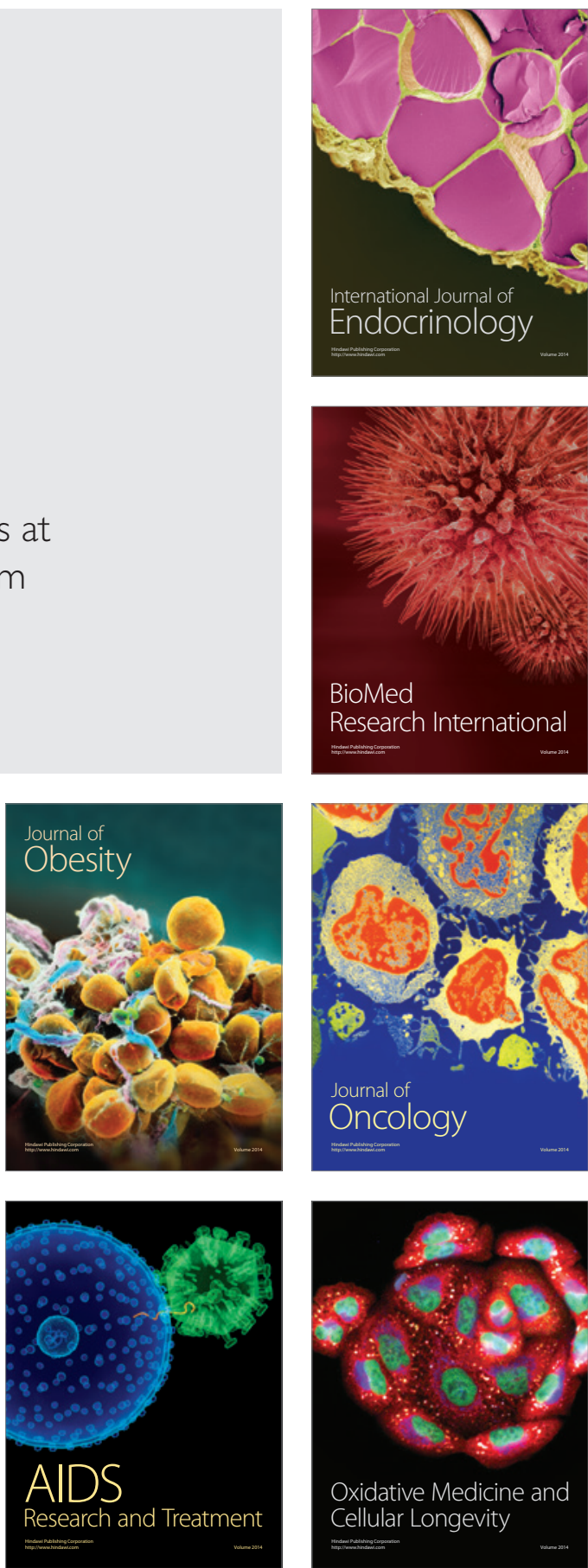